\title{
PARACOCCIDIOIDOMICOSE DE LOCALIZAÇÃO INTRAMEDULAR E CEREBRAL
}

\author{
R. N. MORATO-FERNANDEZ * - P. S. S. BERALDO ** - M. MASINI *** \\ P. H. C. COSTA ****
}

\begin{abstract}
RESUMO - O presente relato de caso, descreve nossa experiência diagnóstica e terapêutica com um paciente que apresentou manifestações clínicas, radiológicas e tomográficas de para coccidioidomicose comprometendo os pulmões, cérebro e, particularmente, a medula espinhal Além da raridade do caso, chama-se a atenção para a extrema dificuldade na elucidação diagnóstica definitiva, obtida somente após laminectomia e biópsia cirúrgica de tumoração intramedular ao nível de C5. O paciente recebeu duas séries de tratamento com, ketoconazol (400 a $600 \mathrm{mg} / \mathrm{dia})$. No início de ambas apresentou crise convulsiva tônico-clônica. Especula-se sobre uma possível resposta inflamatória perigranulomatosa intracerebral, relacionada à intervenção terapêutica, ainda sem relato na literatura.
\end{abstract}

Intra spinal cord and cerebral paracoccidioidomycosis.

SUMMARY - It is reported our diagnosis and therapeutical experience with a patient that presented clinical, radiological and tomographical manifestations of paracoccidioidomycosis with involvement of lungs, brain and spinal cord. Besides being a rare case the authors call attention for the extreme difficulty in defining the final diagnosis, which was achieved only after laminectomy and surgical biopsy of the intra spinal cord tumor at the C5 level. The patient received two series of treatment with ketoconazole (400-600 mg/day). After both the patient presented tonic and clonic convulsive disorders. We speculated about a possible perigranulomatous inflammatory response related to the therapeutical intervention, not yet described in literature.

A paracoccidioidomicose é micose sistêmica provocada pelo Paracoccidioides brasiliensis, de distribuição geográfica definida, restrita ao continente latino-americano. No Brasil incide em proporções significativas, com prevalências variando entre 5,6 e 17,5\%, em provas intradérmicas com paracoccidioidina, como citado por Andrade et al.'. A doença manifesta-se por comprometimento cutaneomücoso, ganglionar e visceral, em que se destacam pulmão, fígado, baço, pâncreas, rim, suprarrenal e osso. O acometimento do sistema nervoso central (SNC) é pouco frequente, variando nas diversas casuísticas entre $9,6 \%$ a $12,5 \%$, predominando no encéfalo 7,15 . As lesões podem apresentar-se tanto na forma meníngea como pseudotumoral (granulomatosa) ou mista. O tratamento tem-se restringido classicamente ao uso de sulfa ou anfotericina B e, mais recentemente, aos derivados imidazólicos 12.

O acometimento intramedular é considerado raro, com apenas três casos registrados na literatura até o momento, nenhum no entanto, associado a lesão cerebral como no presente relato.

Hospital das Doenças do Aparelho Locomotor (HDAL)/Sarah: * Neurologista e Neurofi ᄀ siologista Clínico do HDAL/Sarah; ** Clínico do HDAL/Sarah, Pós-graduando em Clínica Médica, Faculdade de Medicina de Ribeirão Preto/USP; *** Neurocirurgião do HDAL/Sarah; **** Patologista do HDAL/Sarah.

Dr. R. N. Morato-Fernandez - Hospital das Doenças do Aparelho Locomotor (HDAL)/Sarah

Av. W3 Sul, Q. 501, SMHS - 70330 Brasília DF - Brasil 


\section{OBSERVAÇÃO}

GL, trata-se de paciente de 50 anos de idade, do sexo masculino, branco, natural e procedente do Espírito Santo, pedreiro, encaminhado ao HDAL/Sarah em 20-09-88 para reabilitação, com diagnóstico de síndrome de Guillain-Barré. Nessa ocasião, o paciente relatava que há 4 meses havia iniciado a apresentar dor cervical, cãibras generalizadas, seguidas de diminuição progressiva da força em membro inferior (MI) esquerdo (E) e, posteriormente, em MI direito (I>). Evoluiu com piora, passando a apresentar comprometimento dos membros superiores (MS), culminando com alterações sensitivas e motoras nos 4 segmentos. De antecedentes, referia que há 6 anos tinha apresentado lesão ulcerada, comprometendo mucosa labial e língua, cuja biópsia definiu a presença de Paracoccidioides brasiliensis. Informou ainda que, há 1,5 ano apresentou supuração no olho E com diversas tentativas terapêuticas frustas. A biópsia de conjuntiva evidenciou o mesmo agente etiológico. Nas duas ocasiões foi medicado com ketoconazol $(200 \mathrm{mg} / \mathrm{dia})$ por 6 meses e de modo irregular, abandonando o seguimento. Sem relato de crises convulsivas no passado. O exame neurológico na admissão hospitalar evidenciou: consciência preservada, cooperativo, boa articulação da palavra; sem comprometimento dos nervos cranianos; equilíbrio dê tronco comprometido, não sendo possível avaliar a coordenação; fraqueza importante nos MS e MI (vencendo a gravidade, com dificuldade e ausência de movimentos voluntários, respectivamente), além de atrofia muscular intensa; reflexos profundos não obtidos; sinal de Babinski bilateralmente; hipoestesia ao nível de T4 com anestesia abaixo de T6; hipertonia espástica, principalmente nos MI. O quadro clínico era de mielopatia a nível cérvico-torácico. Da rotina laboratorial solicitada chamava atenção: VHS constantemente elevada (49 a $87 \mathrm{~mm} / 1^{*}$ hora), hiper gama e alfa2 globulinemia, mucoproteínas significativamente elevadas (superior a 9,4 mg\%), proteína C reativa positiva, com Látex e FAN negativos; PPD não reator; transaminases e fosfatase alcalina sem alterações; uréia de $90 \mathrm{mg} \%$ e creatinina de $2,1 \mathrm{mg} \%$. O estudo radiológico do tórax mostrou infiltrado intersticial difuso peri-hilar e em ápices, bilateralmente. A eletroneuromiografia dos 4 segmentos mostrou velocidade de condução sensitiva e motora normais. Potencial evocado também nos 4 segmentos foi compatível a defeito da condução no sistema medular sensorial de fibras grossas a nível cervical. Três punções sempre evidenciaram liqüido cefalorraquidiano (LCR) de aspecto translúcido e xantocròmico, com exaustivas pesquisas diretas e culturas para fungos negativas. Os mesmos procedimentos foram realizados na secreção pulmonar obtida por expectoração e punção traqueal, acrescida de pesquisa para BAAR, todas negativas. As análises laboratoriais das amostras de LCR revelaram hipoglicorraquia persistente (até $30 \mathrm{mg} \%$, sendo as glicemias nunca inferiores a $73 \mathrm{mg} \%$ ), proteínas elevadas (até $1260 \mathrm{mg} \%$ ), hipergamaglobulinorraquia (24,2\%) e aumento da atividade de TGO (14 UI) e DHL (54 UI); a reação coloidal de Takata-Ara foi positiva, tipo vermelho; a celularidade mostrou pleocitose (até 20 células/mm3) com predomínio mononuclear; foram realizadas ainda imunofluorescência para sífilis (FTA-Abs), cisticercose, toxoplasmose e esquistossomose, que se revelaram não reagentes. As pesquisas para $P$. brasiliensis, de precipitinas bem como as reações de fixação de complemento (micrométodo, antígeno bruto), imunofluorescência indireta e imunodifusãoi de gel, realizadas no LCR, foram negativas. A mielografia total revelou bloqueio inferior em $\mathrm{Tl}$ e superior em $\mathrm{C} 4$, sugerindo canal estreito em associação com possível lesão intramedular.

Tendo em vista a indefinição do processo etiológico e a forte suspeita clínico-epidemiológica de paracoccidioidomicose, iniciou-se teste terapêutico com derivado imidazólico, ketoconazol (400 $\mathrm{mg} / \mathrm{dia})$ por 30 dias. No quarto dia de tratamento o paciente apresentou crise convulsiva tipo tônico-clónica, associada a intensa bradicardia, que perdurou por três dias, sem evidência de papiledema e com resolução espontânea. Ao término dos 30 dias do tratamento proposto, o paciente apresentava discreta, e transitória melhora do quadro neurológico, com movimentos voluntários nos MI, surgindo ginecomastia e mantendo a função hepática íntegra. Nesta ocasião, em 28-03-89, com o paciente ainda sem diagnóstico etiológico definido, optou-se por. laminectomia exploradora, confirmando-se a presença de lesão expansiva intramedular ao nível de $\mathrm{C} 4$ a $\mathrm{Tl}$ (Fig. 1), tendo a histopatologia da biópsia superficial da piamáter evidenciado processo inflamatório granulomatoso com extensa necrose, pela coloração de Grocott confirmando-se a presença de P. brasiliensis (Fig. 2). Dessa forma, reiniciamos ketoconazol, 17 dias após a conclusão do esquema anterior, na dosagem de $600 \mathrm{mg} / \mathrm{dia}$. No terceiro dia apresentou novo episódio convulsivo, sendo submetido a tomografia computadorizada (TC) de crânio e medula cervical que demonstraram, respectivamente, lesão nodular única na região frontal do hemisfério cerebral $\mathrm{D}$ com discreta captação do contraste (Fig. 3) e moderado alargamento simétrico do cordão medular de C4 a C6. O paciente vem evoluindo com discreta mas progressiva melhora do quadro neurológico e alteração do padrão radiológico pulmonar para estrias de fibrose nos lobos pulmonares superiores, com discretas áreas de consolidação acinares de permeio. Tendo em vista a gineço- 


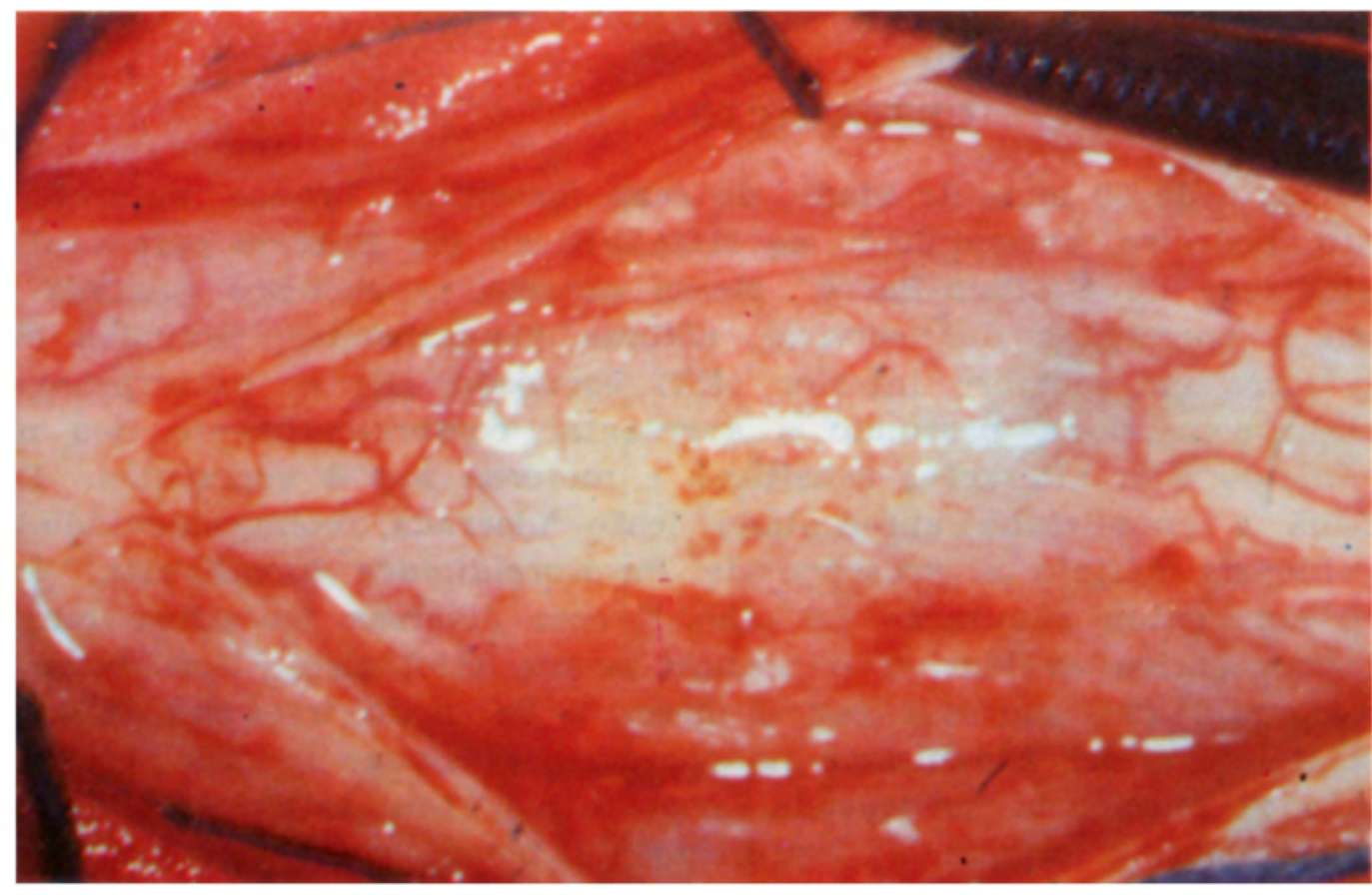

Fig. 1 - Aspecto cirúrgu da medula, apos laminectomia de C4, C5, C6 e C7 $e$ abertura da dura: observa-se tumoração intramedu. lar ao nivel de C5, amarelada, de aspecto cicatricial, tendo sido realizada apenas biopsia superficial.

Fig. 2 - Fotomicrografia da biopsia cirúrgica medular, com coleração de Grocott: observam-se numeroas estruturas arrendondadas, regulares, de tamanhos diversos, por vezes em processo de brotamento, compativeis a $\mathrm{P}$. brasiliensis.
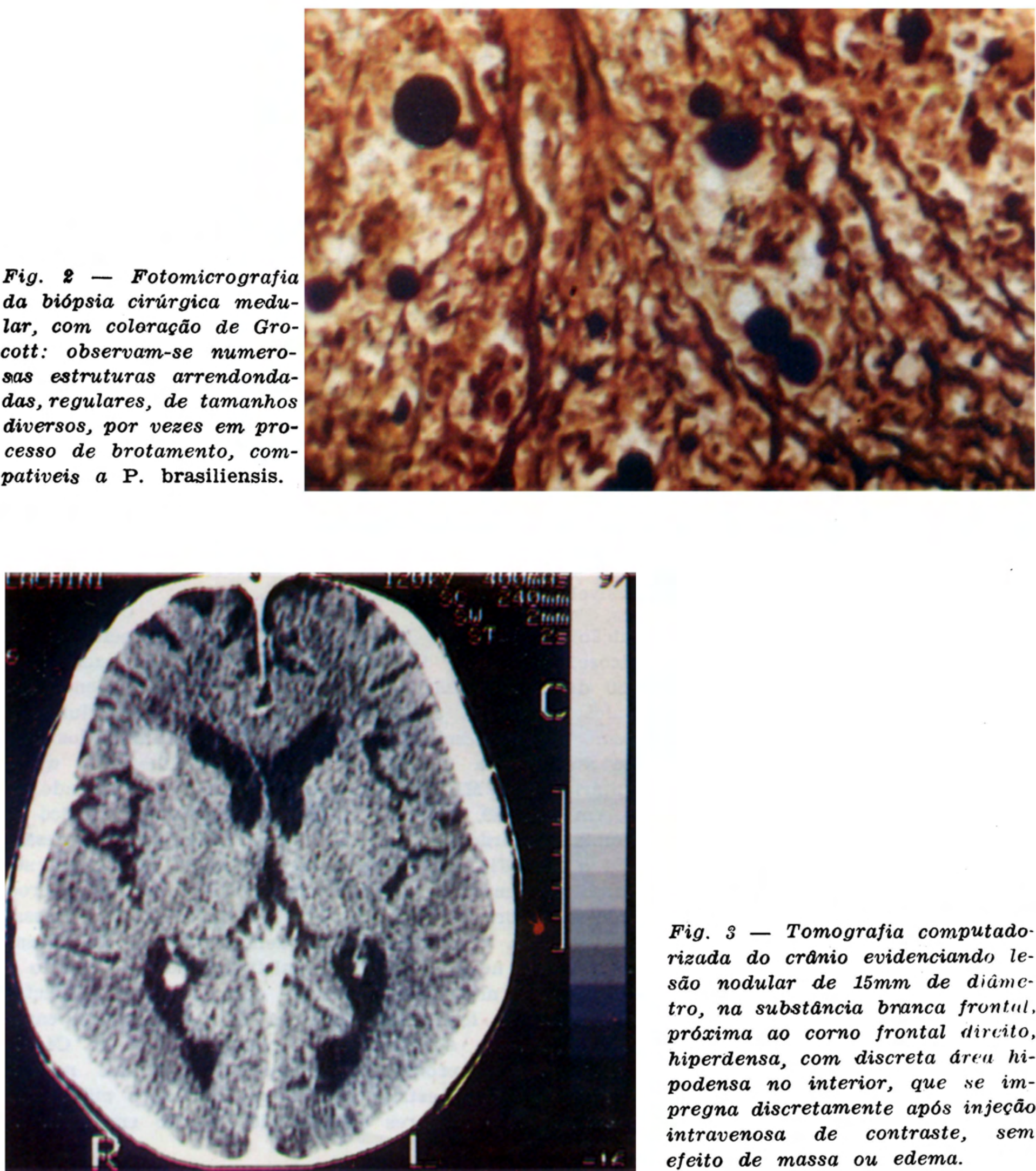

Fig. 3 - Tomografia computado rizada do cranio evidenciando le são nodular de $15 \mathrm{~mm}$ de diâme. tro, na substancia bnanca frontul. proxima ao corno frontal direito hiperdensa, com discreta area hipodensa no interior, que se impregna discretamente apos injeçâo intravenosa de contraste, sem efeito de massa ou edema. 
mastia e apesar da melhora clínica, optou-se por manter tratamento com sulfadoxina 2g/semana. Após 8 meses de seguimento ambulatoria!, o paciente persiste com incontinência urinária, paraparesia, fraqueza nos MS, tendo desaparecido a ginecomastia.

\begin{tabular}{|c|c|c|c|c|c|}
\hline $\begin{array}{l}\text { Autores } \\
\text { (ano) }\end{array}$ & $\begin{array}{l}\text { Dados do } \\
\text { paciente, } \\
\text { origem }\end{array}$ & $\begin{array}{c}\text { Clínica } \\
\text { neurológica }\end{array}$ & 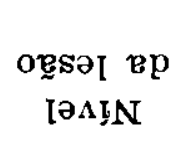 & $\begin{array}{c}\text { Lesões } \\
\text { associadas }\end{array}$ & $\begin{array}{l}\text { Tratamento } \\
\text { e evolução }\end{array}$ \\
\hline $\begin{array}{l}\text { Braga e } \\
\text { Okamura } \\
(1973)\end{array}$ & $\begin{array}{l}\text { masculino, } \\
45 \text { anos, } \\
\text { São Paulo }\end{array}$ & $\begin{array}{l}\text { fraqueza MID, } \\
\text { perda controle } \\
\text { da micção }\end{array}$ & $\mathrm{T} 11-\mathrm{T} 12$ & $\begin{array}{l}\text { pulmōes, } \\
\text { adenopatia } \\
\text { mediastinal }\end{array}$ & $\begin{array}{l}\text { anfotericina, } \\
\text { silfadiazina, } \\
\text { melhora }\end{array}$ \\
\hline $\begin{array}{l}\text { Pedro } \\
\text { et al. } \\
\text { (1980) }\end{array}$ & $\begin{array}{l}\text { masculino, } \\
34 \text { anos, } \\
\text { São Paulo }\end{array}$ & $\begin{array}{l}\text { distúrbio de } \\
\text { sensibilidade } \\
\text { MMII, retenção } \\
\text { urinária }\end{array}$ & T5-T6 & $\begin{array}{l}\text { laringe, } \\
\text { pulmões, } \\
\text { mucosa } \\
\text { oral }\end{array}$ & $\begin{array}{l}\text { anfotericina, } \\
\text { sulfadiazina, } \\
\text { paraplegia } \\
\text { espástica }\end{array}$ \\
\hline $\begin{array}{l}\text { Marchiori } \\
\text { et al. } \\
\text { (1989) }\end{array}$ & $\begin{array}{l}\text { masculino, } \\
51 \text { anos, } \\
\text { Rio de } \\
\text { Janeiro }\end{array}$ & $\begin{array}{l}\text { dor pescoço, } \\
\text { queimação no MIE, } \\
\text { hemiplegia } \\
\text { espástica D e } \\
\text { hipoestesia E }\end{array}$ & $\mathrm{C} 4-\mathrm{C} 5$ & pulmões & $\begin{array}{l}\text { anfotericina, } \\
\text { obito }\end{array}$ \\
\hline
\end{tabular}

Tabela 1 - Casos de lesão intramedular causada pela paracoccidioitomicose registrados na literatura, destacando-se os dados do paciente, quadro neurológico, nivel da lesão, leszes associadas, tratamento e evoluçāo.

MIE, membro inferior esquerdo; MMII, membros inferiores; MID, membro inferior direito.

\section{COMENTÁRIOS}

A paracoccidioidomicose pode acometer o SNC por reação granulomatosa das leptomeninges, ou por granulomas solitários ou múltiplos, comumente localizados nos hemisférios cerebrais, com zonas de necrose em que se identifica o fungo. O primeiro caso comprovado de envolvimento central foi descrito por Maffei 8, em 1943, sob a forma meningítica e de evolução fulminante. Mas já em 1919, Pereira e Jacobs $1^{4}$ tinham chamado a atenção para a possível localização da micose no sistema nervoso, relatando o caso de um paciente jovem com forma cutâneo-ganglionar da paracoccidioidomicose, falecido após quadro epiléptico tipo Bravais-Jacksoniano. Sabe-se hoje que, a partir de lesões primitivas, localizadas geralmente na mucosa bucofaríngea, os parasitos ganham a via linfática e, ulteriormente, a sanguínea, distribuindo-se aos mais diversos setores do organismo. Além do cérebro, foram descritos granulomas no bulbo, ponte, cerebelo, tálamo e raízes espinhais 15 . Até o presente, foram descritos na literatura médica somente três pacientes com lesão intramedular pelo $P$. brasiliensis (Tabela 1). Os casos relatados ocorreram em homens, entre os 34 e 51 anos de idade, acometendo a medula de $\mathrm{C} 4$ a T12 e manifestando-se por fraqueza e distúrbios sensitivos em membros; o acometimento pulmonar concomitante estava presente em todos, seguido do ganglionar e mucoso; em todos os pacientes a mielografia evidenciou o nível da lesão, indicando a laminectomia exploradora e biópsia que confirmou o diagnóstico. A experiência terapêutica se restringiu a anfotericina $B$ e sulfa: um paciente apresentou melhora, outro permaneceu com paraplegia espástica e o outro veio a falecer com complicação relacionada a anfotericina B Farage et al.5 apresentaram um caso similar, porém de localização intradural, extramedular.

$O$ presente relato se assemelha aos demais registrados na literatura, notadamente no que se refere à forma da apresentação clínica e dificuldade diagnostica, diferindo no entanto quanto à concomitância do granuloma intracerebral, demonstrado na TC de crânio e a experiência terapêutica com ketoconazol. O perfil epidemiológico exibido pelo nosso paciente se enquadra naquele traçado por Marques et al. ${ }^{1 * *}$ para pacientes com paracoccidioidomicose: o homem mostrou-se mais acometido (relação 
homem-mulher de 10,7:1); 54\% dos pacientes apresentavam idade entre 35 e 54 anos; os lavradores e pedreiros eram as categorias profissionais mais acometidas.

Do ponto de vista do diagnóstico de envolvimento do SNC na paracoccidioidomicose, os exames complementares, inclusive do LCR, são inespecíficos. O encontro do parasito, assim como a positividade das reações de fixação de complemento e de precipitação, são raramente verificados 16. São descritos, no LCR aumento do teor de gamaglobulina, pleocitose com predomínio de linfócitos e hipoglicorraquia. A natureza da afecção só tem sido suspeitada quando da presença de lesões paracoccidióideas conhecidas em outros órgãos, invariavelmente o pulmonar. Como no presente caso, a TC tem-se revelado útil no reconhecimento do comprometimento encefálico, demonstrando a presença das lesões mesmo antes do surgimento das alterações neurológicas, caracterizadas por estruturas nodulares únicas ou múltiplas, sem sinais de neoformação ou destruição óssea, pequena área de edema perifocal, discreto efeito de massa e, usualmente, concentrando-se o contraste na periferia da lesão $n$.

A experiência clínica 17 e experimental em modelos animais 6 com ketoconazol, nas diversas formas não-nervosas da paracoccidioidomicose, têm sido bastante encorajadoras face aos resultados, facilidade de administração por via oral e relativa inocuidade. Dentre as desvantagens das demais opções terapêuticas, citadas por Negroni 12, sabe-se que as sulfonamidas devem ser administradas por períodos de tempo prolongados ( 3 a 5 anos) e usualmente levam a resistência microbiana secundária, enquanto a anfotericina $B$, além da marcada toxicidade, deve ser administrada por via intravenosa profunda por breves períodos de tempo, com relatos de recidiva após sua interrupção. Nas demais micoses do SNC, o ketoconazol tem exibido resultados também satisfatórios, em doses elevadas $\left(800 \mathrm{mg} / \mathrm{dia}\right.$ em média) e com penetração adequada no LCR ${ }^{3}$. Especificamente na neuroparacoccidioidomicose, o ketoconazol foi utilizado em poucas ocasiões, mas com os mesmos resultados animadores 19. Estas considerações nos levaram a insistir no esquema de ketoconazol para o paciente, em que pese as duas experiências prévias relatadas quando do acometimento mucocutâneo. Os episódios convulsivos apresentados no início ( 3 a 4 dias) das duas séries com ketoconazol, nunca antes descritos especificamente nestas condições, sugerem a possibilidade de resposta inflamatória perigranulomatosa intracerebral ao fungo em destruição, similar ao relatado na neurocisticercose tratada com praziquantel ${ }^{4}$. Considerando o surgimento da ginecomastia, que na série de Sugar et al.is figurou em $21 \%$ dos pacientes tratados com altas doses de ketoconazol $(400$ a $2000 \mathrm{mg})$, optamos por manter o paciente em uso prolongado com sulfadoxina.

Além da raridade do caso, salientamos que, do ponto de vista diagnóstico, o acometimento do SNC pela paracoccidioidomicose depende muito mais de um alto grau de suspeição, com base nos dados epidemiológicos (procedência, sexo, idade, profissão, dentre outros) e clínicos do paciente. O exame neurológico detalhado se impõe em todos os pacientes com paracoccidioidomicose, independentemente da referência de alterações para este lado, considerando-se a gravidade da condição e a possibilidade de, com o tratamento precoce, evitar o surgimento de sequelas incapacitantes. Os exames laboratoriais complementares são de maneira geral inespecíficos, porém indicativos. A propedêutica radiológica (TC, mielografia ou ressonância nuclear magnética) deverá sempre trazer maior subsídio ao esclarecimento do envolvimento encefálico ou intramedular. Do ponto de vista terapêutico o ketoconazol parece demonstrar uma opção, sendo os efeitos colaterais relacionados a esta droga em sua maioria reversíveis com sua suspensão ${ }^{18}$. Os episódios convulsivos observados sugerem a necessidade de melhor conhecimento dos mecanismos de ação dos derivados imidazólicos na neuroparacoccidioidomicose, indagando-se assim a necessidade de uma co-intervenção com corticosteróides, cujos benefícios já foram demonstrados em reações semelhantes como na neurocisticercose tratada com praziquantel 4.

Agradecimento - Agradecemos a Luzimar Gomes de Paiva, Bibliotecária do Instituto Nacional de Medicina do Aparelho Locomotor, pela inestimável colaboração na revisão da bibliografia consultada durante a condução do presente caso. 


\section{REFERÊNCIAS}

1. Andrade JAF, Andrade TM, Lacaz CS, Rodrigues MC, Preuss M, Lourenço R, Badaró R. Inquérito com paracoccidioidina em uma população da Bahia (Brasil). Rev Inst Med Trop São Paulo 1984, 26 : 1.

2. Braga FM, Okamura M. Blastomicose medular. Seara Med Neurocir (São Paulo) 1973, $1: 435$

3. Craven PC, Graybill JR, Jorgensen JH. Dismukes WE, Levine BE. High-dose ketoconazole for treatment of fungal infections of the central nervous system. Ann Intern Med $1983,98: 160$.

4. deGhetaldi LD, Norman RM, Douville AW Jr. Cerebral cysticercosis treated biphasically with dexamethasone and praziquantel. Ann Intern Med 1983, 99 : 179.

5. Farage MF, Braga MRG, Kuhn MDS. Granuloma blastomicótico em medula espinhal. Arq Neuro-Psiquiat (São Paulo) 1977, $35: 151$

6. Finquelievich JL, Gosis AS, Negroni R. Tratamiento con ketoconazole de La paracoccidioidomicosis diseminada de la rata. Rev Argent Micol 1985, 8:6.

7. Hutzler RU, Brussi MLP, Capitani CM, Lima SS. Acometimento neurológico da paracoccidioidomicose avaliado pela tomografia computadorizada de crânio. Rev Paul Med 1985, 103:243.

8. Maffei WE. Micoses do sistema nervoso. An Fac Med TJniv São Paulo 1943, $29: 207$.

9. Marchiori E, Freitas MAL, Ranufo AML. Paracoccidioidomicose medular: relato de um caso. Arq Neuro-Psiquiat (São Paulo) 1989, 47:224.

10. Marques SA, Franco MF, Mendes RP, Silva NCA, Baccili C, Curcelli ED. Eracin ACM, Oliveira CS, Tagliarini JV, Dillon NL. Aspectos epidemiológicos da paracoccidioidomicose na área endêmica de Botucatu (São Paulo - Brasil). Rev Inst Med Trop São Paulo $1983,25: 87$

11. Minghetti G. Tomografia computadorizada dos granulomas blastomicóticos encefálicos Rev Inst Med Trop São Paulo 1983, 25 : 99.

12. Negroni R. Azole derivatives in the treatment of paracoccidioidomycosis. Ann NY Acad Sci 1988, 544:497.

13. Pedro RJ, Branchini MLM, Lucca RS, Silveira ML, Facure NO, Amato V Neto. Paracoccidioidomicose do sistema nervoso central: a propósito de dois casos. Rev Inst Med Trop São Paulo 1980, 22 : 269

14. Pereira JM, Jacobs F. Um caso de blastomicose cutânea com acessos epilépticos. Ann Paul Med Cir 1919, 10:217.

15. Pereira WC, Raphael A, Sallum J. Lesões neurológicas na blastomicose sul-americana. Arq Neuro-Psiquiat (São Paulo) 1965. 23:95.

16. Raphael A. Localização nervosa da blastomicose sul-americana. Arq Neuro-Psiquiat (São Paulo) 1966, 24 :69.

17. Restrepo A, Gomez I, Cano LE, Arango MD, Gutierrez F, Sanin A, Robledo MA Treatment of paracoccidioidomycosis with ketoconazole: a three-year experience. Am J Med 1983, 78:48.

18. Sugar AM, Alsip SG, Galgiani JM, Graybill JR, Dismukes WE, Cloud GA, Cravan PC, Stevens DA. Pharmacology and toxicity of high-dose ketoconazole. Antimicrob Agents Chemother 1987, 31:1874

19. Turovetzky A, Koren F, Negroni R, Grun D, Stortini R. Lesión expansiva de cerebelo por Paracoccidioides brasiliensis. Rev Argent Micol 1980, 3 : 9. 